\section{Fatores de experiência linguística associados à proficiência autoavaliada por usuários de inglês como língua adicional}

\section{Language experience factors associated to self-reported proficiency by users of English as an additional language}

Ana Paula Scholl ${ }^{1}$, Ingrid Finger ${ }^{2}$, Ana Beatriz Arêas da Luz Fontes ${ }^{3}$
Mestre em Linguística Aplicada pela Universidade Federal do Rio Grande do Sul, bolsista CAPES. E-mail: anapaulascholl@gmail.com Docente da Universidade Federal do Rio Grande do Sul, Doutora pela Pontifícia Universidade Católica do Rio Grande do Sul.

E-mail: finger.ingrid@gmail.com do Sul, Doutora pela Universidade do Texas em El Paso.

E-mail ana.fontes@ufrgs.br
RESUMO: Pesquisadores sugerem que o aprendizado de línguas adicionais e a experiência com o uso dessas línguas tem um papel importante no desenvolvimento da competência linguística de um indivíduo (MARIAN, BLUMENFELD e KAUSHANSKAYA, 2007). 0 objetivo do presente estudo foi investigar quais fatores relacionados a uma experiência linguística são associados à proficiência autoavaliada pelos usuários de uma língua adicional. Para esse fim, correlacionamos variáveis da experiência linguística de 535 participantes brasileiros falantes de inglês como língua adicional com a proficiência reportada por eles no Questionário de Experiência e Proficiência Linguística (QuExPLi). Os resultados sugerem que os fatores relacionados a marcos de idade, tempo de imersão e uso atual da língua associam-se de forma significativa à proficiência autorreportada pelos participantes.

Palavras-chave: Experiência linguística; Autoavaliação de proficiência; Questionário de histórico da linguagem.

ABSTRACT: Researchers suggest that learning additional languages and the experience of using them have an important role in the development of the linguistic competence of an individual (MARIAN,; BLUMENFELD, \& KAUSHANSKAYA, 2007). The goal of this study was to investigate which language experience factors are associated with the self-reported proficiency of additional language users. For this purpose, we correlated language experience variables of 535 Brazilian participants, speakers of English as an additional language, with their self-reported proficiency on the Questionário de Experiência e Proficiência Linguística (QuExPLi). Results suggest that the factors related to age, time of immersion, and current use of the language are associated to participants' self-reported proficiency.

Keywords: Language experience; Self-reported proficiency; Language background questionnaire. 


\section{Introdução}

esquisas realizadas visando investigar diferentes aspectos e efeitos cognitivos (atenção, controle inibitório e consciência metalinguística, por exemplo) do uso de mais de uma língua na vida diária de indivíduos vêm obtendo resultados contrastantes (ADESOPE, THOMPSON e URGERLEIDER, 2010; PAAP; GREENBERG, 2013; BRUIN, TRECCANI e DELLA SALA, 2014). Ou seja, há evidências que sugerem que o bilinguismo acarreta variados benefícios cognitivos (BIALYSTOK e MARTIN, 2004; COSTA, HERNÁNDEZ e SEBASTIÁN-GALLÉS, 2008; BIALYSTOK, CRAIK e LUK, 2012), enquanto que outros estudos reportam efeitos nulos, mistos ou negativos em relação ao uso diário de duas línguas ou mais (PAAP; LIU, 2013).

Um possível motivo para a divergência entre as pesquisas em relação aos efeitos cognitivos do uso de duas ou mais línguas na vida diária é o fato de que há uma discrepância muito grande entre os participantes selecionados para esses estudos (GROSJEAN, 2006). Essas inconsistências entre os participantes dos estudos são acentuadas pela falta de instrumentos uniformes para a avaliação de aprendizes de línguas adicionais e bilíngues. Segundo Luk e Bialystok (2013), muitos estudos também carecem de informações mais detalhadas sobre a experiência linguística dos participantes, especialmente sobre a idade de aquisição das línguas, os contextos de uso e a proficiência.

Um instrumento muito utilizado em pesquisas com usuários de línguas adicionais e bilíngues são questionários de histórico da linguagem. Através desses instrumentos busca-se conhecer e entender como os falantes adquiriram e usam as suas línguas e qual a proficiência desenvolvida nelas. Para que tenhamos um questionário que avalie de forma confiável os participantes, é necessário que investiguemos quais fatores que influenciam a experiência bilíngue e a proficiência.
De acordo com a literatura (GROSJEAN, 2013; TREMBLAY, 2011), os critérios que demonstram ter uma maior influência na experiência dos usuários de línguas adicionais e falantes bilíngues são a idade de aquisição das línguas, o tempo de imersão na língua adicional e o uso atual da língua feito pelos falantes.

O fator 'idade' é um tópico com bastante visibilidade em pesquisas sobre aquisição de línguas adicionais. Geralmente fala-se em 'idade de aquisição' ou 'idade de início' (age of onset) para referir-se à idade cronológica com a qual um indivíduo começou a ser exposto a uma língua, que não é a sua língua nativa, de forma significativa (GRANENA e LONG, 2013). A 'idade de início do uso ativo' de uma língua refere-se à idade com que os participantes começaram a usar a sua língua adicional diariamente (LUK e BIALYSTOK, 2013). Segundo Hammers e Blanc (2000), a idade de aquisição desempenha um papel fundamental na construção da experiência linguística de um indivíduo, especialmente no que se refere ao desenvolvimento linguístico, cognitivo, neuropsicológico e sociocultural.

Ao falarmos sobre a idade de aquisição de uma língua adicional, a certeza mais comum que as pessoas têm é a de que as crianças têm mais facilidade de aprender uma língua do que os adultos, concretizada na expressão "quanto mais cedo, melhor". Essa afirmação tem como base pesquisas que mostram que a idade é um fator determinante para o sucesso em uma língua adicional e que quanto mais cedo a idade de início da exposição, mais chances de se obter sucesso na língua (BIALYSTOK, 2001). Pesquisadores que apoiam essa premissa variam em relação ao papel que dão para a idade em determinar esse sucesso, sendo que alguns postulam que ela é o fator mais importante para determinar o desempenho final em uma língua, enquanto outros afirmam que ela é apenas um dos fatores que deve ser levado em consideração.

Pesquisadores relatam correlações negativas entre a idade de aquisição de uma língua adicional e diferentes variáveis linguísticas. Johnson 
e Newport (1989), por exemplo, examinaram o conhecimento de 12 estruturas gramaticais de inglês em uma tarefa de julgamento gramatical de 138 sentenças. Os participantes eram 46 estudantes ou professores de universidades americanas, falantes nativos de chinês ou coreano, que tinham idades entre 3 e 39 anos e deveriam ter tido pelo menos 3 anos de experiência na língua inglesa para participar do estudo. Os pesquisadores encontraram uma correlação negativa forte e linear entre a idade de início da exposição ao inglês (operacionalizada como a idade com a qual os participantes chegaram nos Estados Unidos) e o percentual de acertos na tarefa $(r=-, 77 ; p=<, 01)$, ou seja, quanto mais tarde os participantes haviam chegado nos Estados Unidos, menor a pontuação na tarefa. Além disso, essa correlação foi significativa - e mais robusta - para os participantes que haviam chegado ao país antes dos 15 anos $(r=-, 87 ; p=<, 01)$, mas não para aqueles que chegaram com 17 anos ou mais. Os resultados do estudo de Johnson e Newport (1989) sugerem que os indivíduos que chegaram nos Estados Unidos antes dos 7 anos de idade tiveram uma pontuação equivalente a de falantes nativos e, depois dessa idade, houve um declínio no desempenho dos participantes até a puberdade. Porém, para os que chegaram depois da puberdade, não houve um declínio linear. Esses resultados levaram as autoras a concluir que existem restrições maturacionais para o aprendizado de uma segunda língua.

Em um estudo que sugere uma correlação negativa entre idade e proficiência, Luk e Bialystok (2013) encontraram uma correlação negativa significativa entre a idade início de uso ativo das duas línguas e a proficiência demonstrada pelos participantes nos testes $(r=-, 31 ; p<, 003)$, bem como com a proficiência autoavaliada por eles em produção oral $(r=-, 37$; $p<, 0002)$ e compreensão oral $(r=-, 40 ; p<, 0001)$. Tais resultados indicam que, quanto mais cedo os participantes começaram a usar a língua adicional ativamente, melhor o desempenho deles em testes de proficiência, assim como também maior a sua proficiência autoavaliada em produção e compreensão oral. Segundo as autoras, a idade de início de uso ativo é uma variável mais sensível do que as medidas usuais de idade de aquisição para prever o desempenho em testes e medidas.

De acordo com Singleton (2003), tanto pesquisas quanto observações de quem trata diariamente com aprendizes de uma língua adicional sugerem que começar a aprender uma língua cedo não é estritamente necessário, nem uma condição suficiente, para a obtenção de uma proficiência equivalente à de um falante nativo. Ainda, a literatura sobre bilinguismo precoce sugere que a idade em que um indivíduo tem contato com uma língua pela primeira vez é apenas um dos fatores que determinam a proficiência que será desenvolvida nessa língua.

O tempo de imersão passado em um contexto em que a língua adicional é falada parece influenciar resultados de pesquisas sobre aquisição de segunda língua e bilinguismo (MARIAN et al., 2007). A imersão em uma língua adicional pode se dar em um contexto em que a língua é predominantemente falada, seja ele um país, uma escola, um local de trabalho, ou uma família. Podemos pensar no tempo de imersão também como a 'exposição anterior à língua' ou o 'tempo de exposição à língua', ou seja, o tempo que um indivíduo passou em um ou mais contextos em que a língua é predominantemente utilizada.

Muitos estudiosos adotam o termo 'tempo de residência' (length of residence) como uma variável na área de aquisição de línguas adicionais para operacionalizar a quantidade de exposição de um aprendiz, em um ambiente naturalístico, entre o ponto inicial de contato com a língua adicional e o momento da testagem (MUÑOZ e SINGLETON, 2011). Esse termo é mais encontrado em pesquisas com imigrantes que vivem, no momento da pesquisa, no país em que a sua língua adicional é falada. De acordo com Flege e Liu (2001), o tempo de residência serve como um índice da quantidade de input recebida. Em outras palavras, acredita-se que o tempo de residência 
em um ambiente em que a língua adicional é predominantemente falada é correlacionado positivamente com a quantidade de input na língua que um indivíduo tenha recebido.

O uso que um participante faz da língua adicional no momento em que dada pesquisa é realizada é geralmente medido através de perguntas sobre quais línguas são usadas para realizar certas tarefas do dia a dia, qual a porcentagem do tempo que o participante usa cada uma de suas línguas e a quantidade de horas (em uma base diária ou semanal) que ele as usa para realizar diferentes atividades. Muñoz e Singleton (2011) percebem uma melhoria metodológica nos estudos sobre línguas adicionais em relação às medidas de uso atual das línguas. Segundo os autores, geralmente apenas o tempo de residência ou exposição à língua era levado em consideração, porém, estudos mais recentes utilizam outras medidas do uso que é feito pelos participantes. Essas medidas costumam ser coletadas através de questionários ou entrevistas.

Os resultados de estudos que investigam o uso da língua no momento da pesquisa (MOYER, 2005; DE CARLI et al., 2014) sugerem que os contextos em que uma língua adicional é utilizada e, especialmente, a frequência com que ela é usada para realizar diferentes atividades influenciam a proficiência desenvolvida por um aprendiz. Dessa forma, acreditamos que esse seja um fator importante a ser incluído em um instrumento que avalie a experiência linguística de um indivíduo.

\section{0 presente estudo}

O presente estudo teve como objetivo investigar quais fatores de uma experiência linguística são associados à proficiência autoavaliada em uma língua adicional. Para esse fim, correlacionamos as respostas dos participantes do presente estudo em relação a marcos de idade, tempo de imersão e uso atual da língua com a proficiência reportada por eles nas quatro habilidades - compreensão leitora, compreensão oral, produção escrita e produção oral - no Questionário de Experiência e Proficiência Linguística (QuExPLi), adaptado de Scholl e Finger (2013).

Com base na literatura citada anteriormente (MARIAN, BLUMENFELD e KAUSHANSKAYA, 2007; LUK e BIALYSTOK, 2013), esperava-se uma associação dos fatores referentes a marcos de idade, tempo de imersão e uso atual da língua com a proficiência reportada no questionário pelos participantes nas quatro habilidades - compreensão leitora, compreensão oral, produção escrita e produção oral - havendo certa variabilidade em relação às habilidades com as quais esses fatores seriam associados.

\subsection{Participantes}

O questionário foi administrado para 535 estudantes da UFRGS ( $M=25$ anos, $\mathrm{SD}=6$; 250 mulheres, 285 homens). Os participantes foram recrutados ao realizarem o TOEFL ITP proporcionado pela universidade, com o fim de ingressar no programa Ciência sem Fronteiras, nos cursos presenciais do Idiomas sem Fronteiras ou obter certificação de proficiência na língua inglesa.

Os participantes eram todos brasileiros, tendo o português como primeira língua. Todos classificaram a língua inglesa como sendo sua segunda (L2) ou terceira língua (L3), de acordo com a idade de aquisição. Alguns participantes classificaram outra língua como L2 e inglês como L3, pois muitos tiveram espanhol na escola e logo depois começaram a aprender a língua inglesa. Informações sobre o histórico de aquisição e uso da língua inglesa reportadas pelos participantes podem ser encontradas na Tabela 1. 
Tabela 1 - Histórico de aprendizagem e uso do inglês reportado pelos participantes no QuExPLi (Estudo 1)

\begin{tabular}{|c|c|c|}
\hline \multirow{2}{*}{ Medidas de histórico da linguagem } & \multicolumn{2}{|c|}{ Língua adicional (inglês) } \\
\hline & M & DP \\
\hline \multicolumn{3}{|l|}{ Marcos de idade (anos) } \\
\hline Começou a aprender & 10,5 & 3,9 \\
\hline Começou a usar ativamente & 16,0 & 4,6 \\
\hline Tornou-se fluente & 18,3 & 4,4 \\
\hline \multicolumn{3}{|l|}{ Contribuição para o aprendizado* } \\
\hline da família & 1,1 & 2,2 \\
\hline de amigos & 2,9 & 1,8 \\
\hline da leitura & 5,0 & 3,0 \\
\hline de textos acadêmicos & 4,4 & 1,8 \\
\hline de televisão/filmes & 4,4 & 1,8 \\
\hline da música & 4,9 & 1,4 \\
\hline da internet & 5,2 & 1,2 \\
\hline de curso de línguas & 4,6 & 2,1 \\
\hline \multicolumn{3}{|l|}{ Imersão (meses) } \\
\hline Em um país & 2,4 & 8,4 \\
\hline Em uma família & 4,3 & 29,5 \\
\hline Em uma escola ou trabalho & 23,8 & 43,9 \\
\hline \multicolumn{3}{|l|}{ Frequência** } \\
\hline Fala com o pai & 1,3 & 1,2 \\
\hline Fala com a mãe & 1,3 & 1,3 \\
\hline Fala com familiares & 1,6 & 1,3 \\
\hline Fala com amigos & 2,9 & 1,5 \\
\hline Fala na escola/trabalho & 3,1 & 1,7 \\
\hline Escreve na escola/trabalho & 4,1 & 1,6 \\
\hline Tempo diário usando a língua (\%) & 16,1 & 16,3 \\
\hline \multicolumn{3}{|l|}{ Horas diárias } \\
\hline TV/filmes & 1,7 & 1,5 \\
\hline Música & 2 & 2,2 \\
\hline Videogames & 0,7 & 1,7 \\
\hline Leitura geral & 1,2 & 1,9 \\
\hline Leitura acadêmica & 1,5 & 2 \\
\hline Escrever & 0,8 & 1,3 \\
\hline Falar & 0,8 & 1,5 \\
\hline
\end{tabular}

* Escala de 0 (nada) a 6 (muito).

** Escala de 1 (algumas vezes por ano) a 6 (diariamente).
A idade de aquisição da língua inglesa pelos participantes variou entre 2 e 42 anos ( $M=10,5$ anos, $D P=3,9)$. Todos os participantes aprenderam a língua inglesa depois da língua portuguesa. Os participantes relataram terem começado a usar ativamente a língua entre os 4 e os 42 anos $(M=16$ anos, $\mathrm{DP}=4,6)$. A idade com a qual os participantes relataram terem se tornado fluentes em inglês variou entre 4 e 35 anos ( $M=18,3$ anos; $D P=4,4)$.

A maioria dos participantes aprendeu a língua inglesa na escola, em cursos de línguas ou sozinhos. Ao reportarem os diferentes fatores que contribuíram para o aprendizado da L2, os participantes declararam que os fatores predominantes foram assistir à televisão/filmes e usar a internet, seguidos por leitura e música.

A média de tempo que os participantes relataram ter passado em um país em que a língua inglesa é falada foi de 2.5 meses ( $D P=8,4)$, enquanto que a média de tempo passado em uma família falante da L2 foi de 4,4 meses $(\mathrm{DP}=29,5)$. Os participantes relataram ter vivenciado uma média de 23,9 meses ( DP $=44$ ) em uma escola/universidade ou trabalho em que a língua é falada. No presente momento, a porcentagem média de tempo em que o inglês é usado diariamente segundo os participantes é de 16,1\% (DP=16,3).

\subsection{Materiais e procedimentos}

O questionário utilizado no presente estudo foi adaptado de Scholl e Finger (2013). O questionário contém perguntas sobre o histórico das línguas dos participantes, ou seja, perguntas sobre quais línguas os falantes sabem, quando, como e onde essas línguas foram adquiridas e o tempo de exposição às línguas em diferentes contextos. 0 questionário também abarca questões sobre quais línguas são usadas em quais contextos, com qual propósito e frequência. O questionário inclui uma escala de autoavaliação de proficiência em leitura, escrita, compreensão oral e fala, na qual os participantes devem avaliar a sua proficiência em uma escala de 1 a 6 , sendo $1=$ muito baixo e 6 = proficiente. Por último, há um espaço para informações adicionais. 
O questionário foi entregue aos participantes enquanto eles aguardavam o início da prova. Ao chegar para realizar a prova, os participantes recebiam o QuExPLi e assinavam o Termo de Consentimento Livre e Esclarecido relativo ao projeto de número CAAE 37252514.5.0000.5347. Na sala em que realizariam o TOEFL ITP, junto com os demais participantes, os alunos respondiam o questionário antes que a prova tivesse início. 0 preenchimento do questionário levava entre 10 e 15 minutos e os instrumentos eram entregues para os aplicadores da prova antes que ela iniciasse.

\subsection{Análise}

As respostas dos participantes nos questionários foram inseridas manualmente em uma planilha no Excel, que foi exportada para o SPSS (versão 18.1), software no qual as análises estatísticas foram realizadas. Os fatores idade de aquisição, idade de início de uso ativo, idade de fluência, meses em um país, meses em uma família e meses em uma escola ou trabalho em que a língua é falada e o tempo diário (frequência, porcentagem e número de horas) em que os participantes reportaram usar a língua foram correlacionadas com as quatro habilidades autoavaliadas no questionário compreensão leitora, produção escrita, compreensão oral e produção oral. Também correlacionamos as quatro habilidades entre si.

\section{Resultados e discussão}

Primeiramente, correlacionamos a proficiência reportada pelos participantes nas quatro habilidades entre si. As habilidades de compreensão leitora e de compreensão oral correlacionaram-se positivamente $(r=, 722$; $p<, 01$ ). Da mesma forma, as habilidades de compreensão leitora e de produção escrita correlacionaram-se positivamente de forma significativa $(r=, 729$; $p<, 01)$. As habilidades de compreensão leitora e de produção oral também se correlacionaram positivamente $(r=, 681 ; p<, 01)$. Em concordância, as habilidades de produção escrita e de compreensão oral $(r=, 707$; $p<, 01)$ e de produção escrita e de produção oral $(r=, 795 ; p<, 01)$ apresentaram correlações positivas significativas. As habilidades de compreensão oral e de produção oral, por sua vez, também se correlacionaram de forma positiva $(r=, 791 ; p<, 01)$. Dessa forma, todas as habilidades autoavaliadas correlacionaram-se de forma positiva e significativa entre si.

As correlações positivas fortes que foram evidenciadas indicam que os participantes mostraram-se consistentes ao reportarem a sua proficiência nas quatro habilidades. Em outras palavras, esses dados indicam que, quando os participantes se avaliaram bem em uma das habilidades, avaliaram-se bem também nas outras. Tais resultados são consistentes com as correlações reportadas por Powers (2010), extraídas das análises de testes de participantes que realizaram o TOEIC, que evidenciaram associações entre o desempenho dos participantes nas quatro habilidades. Dessa forma, esses resultados estão em concordância com a afirmação de Powers (2010), de que o desempenho de usuários de uma língua adicional nas quatro habilidades é altamente relacionado, ou seja, um falante que tem um bom desempenho em compreensão oral tende a ter um bom desempenho em compreensão leitora e produção escrita, por exemplo.

\subsection{Marcos de idade}

Em relação aos marcos de idade, a idade com a qual os participantes começaram a adquirir a língua, a idade com a qual começaram a usar o inglês ativamente e a idade com a qual se tornaram fluentes foram correlacionadas com a proficiência reportada pelos participantes nas quatro habilidades. A idade de aquisição da língua inglesa correlacionou-se negativamente com a proficiência autorreportada nas habilidades de compreensão leitora $(r=-, 279 ; p<, 01)$, produção escrita $(r=-, 269 ; p<, 01)$, compreensão oral 
$(r=-, 308 ; p<, 01)$ e produção oral $(r=-, 265 ; p<, 01)$. A idade em que os participantes relataram ter começado a usar a língua adicional ativamente apresentou uma correlação negativa com a proficiência autorreportada nas habilidades de compreensão leitora $(r=-, 259 ; p<, 01)$, produção escrita $(r=-, 259 ; p<, 01)$, compreensão oral $(r=-, 372 ; p<, 01)$ e produção oral $(r=-, 286 ; p<, 01)$. A idade em que os participantes relataram terem se tornado fluentes em inglês também se correlacionou negativamente com a proficiência reportada nas habilidades de compreensão leitora $(r=-, 252$; $p<, 01)$, produção escrita $(r=-, 165 ; p<, 01)$, compreensão oral $(r=-, 264$; $p<, 01)$ e produção oral $(r=-, 245 ; p<, 01)$. Dessa forma, verificou-se que quanto menor a idade reportada pelos participantes em relação aos marcos de idade, maior a proficiência avaliada por eles nas quatro habilidades.

Os fatores relacionados a marcos de idade tiveram uma correlação negativa significativa com a proficiência reportada nas quatro habilidades, ou seja, quanto antes os participantes adquiriram, começaram a usar e tornaram-se fluentes na língua inglesa, maior foi o nível de proficiência reportado por eles. Esses resultados estão de acordo com Marian et al. (2007) e Luk e Bialystok (2013) que encontraram associações entre os marcos de idade na língua adicional e a proficiência autoavaliada pelos participantes, porém Luk e Bialystok (2013) encontraram apenas correlações significativas entre a autoavaliação nas habilidades de produção oral $(r=-, 37 ; p<, 0002)$ e compreensão oral $(r=-, 40 ; p<, 0001)$ e a idade de uso ativo da língua adicional, enquanto que no presente estudo encontramos correlações significativas entre a variável e a autoavaliação nas quatro habilidades.

\subsection{Tempo de imersão}

Em relação ao tempo de imersão na língua adicional, correlacionamos os meses que os participantes passaram em um país em que o inglês é falado, assim como em uma família e em uma escola ou trabalho, com a proficiência reportada por eles nas quatro habilidades. O número de meses que os participantes passaram em um país em que a língua inglesa é falada correlacionou-se de forma positiva com a proficiência na língua reportada pelos participantes nas habilidades de compreensão leitora $(r=, 147$; $p<, 01)$, produção escrita $(r=, 187 ; p<, 01)$, compreensão oral $(r=, 162$; $p<, 01)$ e produção oral $(r=, 203 ; p<, 01)$. Em outras palavras, quanto mais tempo os participantes passaram em um país de língua inglesa, melhor eles se avaliaram no questionário. A associação positiva entre as variáveis 'meses em um país em que o inglês é falado' e 'proficiência autoavaliada' pode ser relacionada ao fato de que quanto mais tempo os participantes passaram em imersão em um país, mais eles tiveram oportunidades de usar o inglês para diferentes situações do dia a dia, melhorando assim o seu desempenho na língua.

A análise envolvendo o número de meses que os participantes passaram com uma família na qual o inglês é falado revelou uma correlação positiva significativa com as habilidades de compreensão oral $(r=, 131 ; p<, 01)$ e de produção oral $(r=, 118 ; p<, 01)$, ou seja, quanto mais tempo os participantes passaram com uma família falante da língua adicional, melhor se avaliaram nessas habilidades. As correlações entre os meses passados com uma família e as habilidades de compreensão leitora e de produção escrita não foram significativas, $r=, 077$ e $r=, 050$, respectivamente. Essa relação significativa apenas com as habilidades orais pode ter ocorrido devido ao fato de que os participantes têm apenas contato oral em inglês com a família com que conviveram, desenvolvendo essas habilidades de forma mais expressiva.

Já os meses passados em uma escola ou trabalho em que a língua é falada correlacionaram-se positivamente de forma significativa com as habilidades de produção escrita $(r=, 130 ; p<, 01)$ e de produção oral $(r=, 102$; $p<, 05)$, enquanto que as correlações com compreensão leitora e compreensão oral não foram significativas, $r=, 048$ e $r=, 066$, respectivamente. 
Dessa maneira, houve uma associação positiva moderada entre o tempo que os participantes passaram em um ambiente de trabalho ou escolar que utiliza predominantemente o inglês e a autoavaliação da proficiência nas habilidades de produção oral e escrita, ou seja, quanto mais tempo eles passaram nesses ambientes, melhor se avaliaram nas habilidades produtivas.

Tais resultados estão, em parte, em concordância com os resultados encontrados por Marian e colaboradores (2007), que evidenciam que o tempo passado em um trabalho em que a língua adicional é usada previu apenas a proficiência autorreportada na habilidade de produção escrita. No presente estudo, o tempo que os participantes passaram imersos em um trabalho ou escola em que a língua adicional é predominantemente usada correlacionou-se também com a habilidade de produção oral. É possível que essa associação aconteça principalmente entre esses fatores, pois em um local de trabalho a escrita e a fala podem ser mais exigidas do que as outras habilidades, assim como é mais comum que os usuários de uma língua adicional recebam um feedback de professores, chefes ou colega em relação ao desenvolvimento dessas habilidades.

No presente estudo, o tempo passado em um país em que o inglês é predominantemente falado foi a única variável do tempo de imersão na língua que se correlacionou de forma significativa com a proficiência autoavaliada pelos participantes nas quatro habilidades. Essa variável pode ter se correlacionado de forma mais significativa do que as outras referentes ao tempo de imersão - meses passados em uma família e meses passados em uma escola ou trabalho - por ser relacionada a uma experiência mais intensa e variada de uso da língua.

\subsection{Uso atual da língua}

Em relação ao uso atual do inglês que é feito pelos participantes, correlacionamos as respostas nas questões sobre a frequência com a qual a língua é usada para realizar diferentes atividades, sobre o percentual de tempo diário de uso da língua e sobre o número de horas que a língua é usada diariamente com a proficiência autoavaliada pelos participantes. A frequência de fala com o pai na língua adicional correlacionou-se positivamente de forma significativa com as habilidades de produção escrita $(r=, 186$; $p<, 05)$, compreensão oral $(r=, 223 ; p<, 01)$ e produção oral $(r=, 230 ; p<, 01)$ e de forma não significativa com a habilidade de compreensão leitora $(r=, 125)$. A frequência de fala com a mãe teve uma correlação positiva com as habilidades de compreensão leitora $(r=, 179 ; p<, 05)$, produção escrita $(r=, 239 ; p<, 01)$, compreensão oral $(r=, 315 ; p<, 01)$ e produção oral $(r=, 292 ; p<, 01)$. A frequência de fala com outros familiares também se correlacionou significativamente com as habilidades de compreensão leitora $(r=, 194 ; p<, 01)$, produção escrita $(r=, 212 ; p<, 01)$, compreensão oral $(r=, 292 ; p<, 01)$ e produção oral $(r=, 319 ; p<, 01)$. A frequência de fala com amigos teve uma correlação positiva significativa com as quatro habilidades: compreensão leitora $(r=, 194 ; p<, 05)$, produção escrita $(r=, 200 ; p<, 01)$, compreensão oral $(r=, 241 ; p<, 01)$ e produção oral $(r=, 255 ; p<, 01)$. A frequência com que os participantes usam o inglês para falar na escola ou no trabalho também se correlacionou de forma positiva e significativa com as habilidades de compreensão leitora $(r=, 148 ; p<, 05)$, produção escrita $(r=, 187 ; p<, 01)$, compreensão oral $(r=, 193 ; p<, 01)$ e produção oral $(r=, 214 ; p<, 01)$. Por último, a frequência com que os participantes leem e escrevem na escola ou trabalho teve uma correlação positiva significativa com a proficiência reportada nas quatro habilidades: compreensão leitora $(r=, 301 ; p<, 05)$, produção escrita $(r=, 231 ; p<, 01)$, compreensão oral $(r=, 216 ; p<, 01)$ e produção oral $(r=, 178 ; p<, 01)$. As correlações positivas significativas entre as variáveis 'frequência de uso' e 'proficiência autoavaliada' sugerem que quanto maior a frequência de uso do inglês para diferentes atividades melhor os participantes avaliam a sua proficiência. 
Dessa maneira, a frequência com que os participantes usam a língua inglesa para diferentes funções associou-se com a proficiência autorreportada por eles no questionário. As frequências de fala com a mãe, com outros familiares, com os amigos, com que falam na escola ou trabalho e com que leem na escola ou trabalho correlacionaram-se positivamente com as quatro habilidades autoavaliadas. Apenas a frequência de fala com o pai não se associou de forma significativa com as quatro habilidades, pois não se correlacionou com produção leitora. Como uma medida de uso atual da língua, podemos perceber que a maior frequência com que os participantes usam o inglês em diferentes contextos indica uma melhor avaliação na sua proficiência autorreportada.

O percentual de tempo diário que os participantes relataram usar a segunda língua no momento da pesquisa foi correlacionado positivamente de forma significativa com a proficiência autoavaliada nas habilidades de compreensão leitora $(r=, 193 ; p<, 01)$, produção escrita $(r=, 233 ; p<, 01)$, compreensão oral $(r=, 252 ; p<, 01)$ e produção oral $(r=, 226 ; p<, 01)$. Assim, quanto mais tempo os participantes usam inglês diariamente, melhor eles avaliaram a sua proficiência nas quatro habilidades.

Quando medido através do número de horas diárias que os participantes relataram usar a língua inglesa para diferentes atividades (assistir TV/ filmes, ouvir música, jogar videogames, ler livros e revistas, ler textos acadêmicos, escrever e falar), o tempo de uso diário não se correlacionou significativamente com a proficiência autorreportada nas quatro habilidades (compreensão leitora $r=-$,30; produção escrita $r=-, 31$; compreensão oral $r=-, 009$ e produção oral $r=-, 042$ ). Tais resultados não são consistentes com as outras duas medidas de uso atual da língua adicional (frequência de uso em diferentes contextos e percentual de uso diário) em que houve correlações significativas entre o uso e a proficiência autoavaliada. Uma possível explicação para que essa medida não tenha sido significativamente correlacionada com a proficiência reportada pelos participantes no questionário é que eles não tenham conseguido estimar de forma acurada o número de horas que usam a língua inglesa para diferentes atividades, pois as outras duas medidas de tempo de uso atual apresentaram correlações positivas significativas, indicando que quanto mais uso, melhor a proficiência autorreportada.

Dessa forma, os resultados obtidos em relação ao uso atual da língua adicional demonstraram consistência quando avaliados através da frequência com a qual o inglês é usado para realizar diferentes atividades e o percentual de tempo de uso diário da língua, evidenciando correlações positivas significativas moderadas com a proficiência autoavaliada. Já a medida de número de horas que a língua é usada para realizar diversas atividades diariamente não apresentou resultados consistentes com as duas outras medidas de uso atual da língua, pois não se correlacionou de forma significativa com a proficiência autorreportada pelos participantes.

Todas as correlações entre as respostas dadas pelos participantes nas questões referentes à experiência linguística na língua adicional (LA) e a proficiência autoavaliada no Questionário de Proficiência e Experiência Linguística (QuExPLi) são apresentadas na Tabela 2.

A partir dos resultados apresentados, é possível concluir que nossa hipótese de que os fatores referentes a marcos de idade, tempo de imersão e uso atual da língua seriam associados com a proficiência autorreportada no presente estudo, havendo certa variabilidade em relação às habilidades com as quais esses fatores seriam associados, foi confirmada. 
Tabela 2 - Correlações entre experiência linguística e proficiência autoavaliada no QuExPLi

\begin{tabular}{lcccc}
\hline & $\begin{array}{c}\text { Compreensão } \\
\text { leitora }\end{array}$ & $\begin{array}{c}\text { Produção } \\
\text { escrita }\end{array}$ & $\begin{array}{c}\text { Compreensão } \\
\text { oral }\end{array}$ & $\begin{array}{c}\text { Produção } \\
\text { oral }\end{array}$ \\
\hline Idade de aquisição &,$- 279^{* *}$ & -269 &,$- 308^{* *}$ &,$- 265^{* *}$ \\
Idade de uso ativo &,$- 259^{* *}$ &,$- 259^{* *}$ &,$- 372^{* *}$ &,$- 286^{* *}$ \\
Idade fluência &,$- 252^{* *}$ &,$- 165^{* *}$ &,$- 264^{* *}$ &,$- 245^{* *}$ \\
Meses em um país &, $147^{* *}$ &, $187^{* *}$ &, $162^{* *}$ &, $203^{* *}$ \\
Meses em uma família &, 077 &, 05 &, $131^{* *}$ &, $118^{*}$ \\
Meses em uma escola ou trabalho &, 048 &, $130^{* *}$ &, 066 &, $102^{*}$ \\
Frequência que fala com o pai na LA &, 125 &, $186^{*}$ &, $223^{* *}$ &, $230^{* *}$ \\
Frequência que fala com a mãe na LA &, $179^{*}$ &, $239^{* *}$ &, $315^{* *}$ &, $292^{* *}$ \\
Frequência que fala com familiares na LA &, $194^{* *}$ &, $212^{* *}$ &, $292^{* *}$ &, $319^{* *}$ \\
Frequência que fala com amigos na LA &, $194^{* *}$ &, $200^{* *}$ &, $241^{* *}$ &, $255^{* *}$ \\
Frequência que fala na escola/ &, $148^{* *}$ &, $187^{* *}$ &, $193^{* *}$ &, $214^{* *}$ \\
trabalho na LA & & & &, $216^{* *}$ \\
\hline $\begin{array}{l}\text { Frequência que escreve na escola/ } \\
\text { trabalho na LA }\end{array}$ &, $301^{* *}$ &, $231^{* *}$ &, $178^{* *}$ \\
Tempo diário (\%) &, $193^{* *}$ &, $233^{* *}$ &, $252^{* *}$ &, $226^{* *}$ \\
Horas diárias &,- 30 &,- 31 &,- 009 &,- 042 \\
\hline
\end{tabular}

${ }^{*} \mathrm{p}<, 05 ;{ }^{* *} \mathrm{p}<, 01 ; * * * \mathrm{p}<, 001$

\section{Conclusão}

A presente pesquisa surgiu devido à necessidade que há nos campos de aquisição de línguas adicionais e bilinguismo da adoção de instrumentos uniformes para avaliar o histórico das línguas e a proficiência linguística de indivíduos. Com um instrumento que leve em consideração os fatores de uma experiência linguística que se associam à proficiência, é possível ter uma compreensão mais global do desenvolvimento da língua e da proficiência dos participantes em estudos. Assim, a consciência de quais fatores são relacionados à proficiência desenvolvida proporciona ao pesquisador um entendimento mais completo dos seus participantes.

O presente estudo teve como objetivo investigar quais fatores de uma experiência linguística são associados à proficiência autoavaliada em uma língua adicional. Ao analisar os resultados, percebemos que os fatores marcos de idade, tempo de imersão e uso atual da língua correlacionaram-se significativamente, em sua maioria, com a proficiência autoavaliada pelos participantes, indicando uma associação entre esses fatores. Tais resultados sugerem que esses fatores devem ser levados em consideração na avaliação do perfil linguístico dos participantes de pesquisas de aquisição de língua adicional e bilinguismo.

\section{Referências}

ADESOPE, O; LAVIN, T.; THOMPSON, T.; UNGERLEIDER, C. A Systematic review and MetaAnalysis of the Cognitive Correlates of Bilingualism. Review of Educational Research, v. 80, n. 2, p. 207-245, 2010. https://doi.org/10.3102/0034654310368803

BIALYSTOK, Ellen. Bilingualism in Development: Language, Literacy, and Cognition. New York: Cambridge University Press, 2001. https://doi.org/10.1017/CB09780511605963

BIALYSTOK, E.; MARTIN, M. Attention and inhibition in bilingual children: Evidence from the dimensional change card sort task. Developmental Science, v. 7, p. 325-339, 2004 https://doi.org/10.1111/j.1467-7687.2004.00351.x

BIALYSTOK, E.; CRAIK, F.; LUK, G. Bilingualism: consequences for mind and brain. Trends in Cognitive Sciences, v. 16, n. 4, p. 240-250, 2012. https://doi.org/10.1016/j.tics.2012.03.001

BRUIN, A.; TRECCANI, B.; DELLA SALA, S. Cognitive Advantage in Bilingualism: An Example of Publication Bias? Psychological Science, v. 26, n. 1, p. 99-107, 2015.

COSTA, A., HERNÁNDEZ, M., \& SEBASTIÁN-GALLÉS, N. Bilingualism aids conflict resolution: Evidence from the ANT task. Cognition, v. 106, n. 1, p. 59-86, 2008. https:// doi.org/10.1016/j.cognition.2006.12.013

FLEGE, J.; LIU, S. The effect of experience on adults' acquisition of a second language. SSLA, v. 23, p. 527-552, 2001. 
GRANENA, G.; LONG, M. Introduction and overview. In: GRANEMA, G.; LONG, M. (Eds.). Sensitive periods, language aptitude, and ultimate L2 attainment. Language Learning \& Language Teaching 35. Amsterdam: John Benjamins Publishing Company, 2013. p. ix-xiii. https://doi.org/10.1075/1llt.35.002int

GROSJEAN, F. Studying bilinguals: Methodological and conceptual issues. In: BHATIA, T.; RITCHIE, W. (Ed.). The handbook of bilingualism. Malden: Blackwell, 2006. p. 32-63. https://doi.org/10.1002/9780470756997.ch2

GROSJEAN, F. Bilingualism: a short introduction. In: GROSJEAN, F.; LI, P. The Psycholinguistics of Bilingualism. Wiley-Blackwell, 2013. p. 5-21.

JOHNSON, J.; NEWPORT, E. Critical Period Effects in Second Language Learning: The Influence of Maturational State on the Acquisition of English as a second Language. Cognitive Psychology, v. 21, n. 1, p. 60-99, 1989. https://doi.org/10.1016/00100285(89)90003-0

LUK, G.; BIALYSTOK, E. Bilingualism is not a categorical variable: Interaction between language proficiency and usage. Journal of Cognitive Psychology, v. 25, n. 5, p. 605-621, 2013. https://doi.org/10.1080/20445911.2013.795574

MARIAN, V., BLUMENFELD, H. K., \& KAUSHANSKAYA, M. The Language Experience and Proficiency Questionnaire (LEAP-Q): Assessing language profiles in bilinguals and multilinguals. Journal of Speech, Language, and Hearing Research, v. 50, n. 4, p. 940-967, 2007. https://doi.org/10.1044/1092-4388(2007/067)

MOYER, A. Formal and Informal Experiential Realms in German as a Foreign Language: A Preliminary Investigation. Foreign Language Annals, v. 38, n. 3, p. 377-387, 2005. https:// doi.org/10.1111/j.1944-9720.2005.tb02224.x

MUÑOZ, C.; SINGLETON, D. A critical review of age-related research on L2 ultimate attainment. Language Teaching, v. 44, n. 1, p. 1-35, 2011. https://doi.org/10.1017/ S0261444810000327

PAAP, K.; GREENBERG, Z. There is no coherent evidence for a bilingual advantage in executive processing. Cognitive Psychology, v. 66, n. 2, p. 232-258, 2013. https://doi. org/10.1016/j.cogpsych.2012.12.002

PAAP, K.; LIU, Y. Conflict resolution in sentence processing is the same for bilinguals and monolinguals: The role of confirmation bias in testing for bilingual advantages. Journal of Neurolinguistics, v. 27, n. 1, p. 50-74, 2014. https://doi.org/10.1016/j. jneuroling.2013.09.002
POWERS, D. The Case for a Comprehensive, Four-Skills Assessment of English Language Proficiency. ETS: TOEIC Compendium, 2010.

SINGLETON, D. Critical Period or General Age Factor(s)? In: GARCÍA MAYO, M.; GARCIA LECUMBERRI, M. (Eds.). Age and the Acquisition of English as a Foreigner Language. Clevedon: Multilingual Matters, 2003, p. 3-22.

SCHOLL, A. P.; FINGER, I. Elaboração de um questionário de histórico da linguagem para pesquisas com bilíngues. Nonada: Letras em Revista, v. 2, n. 21, 2013.

TREMBLAY, A. Proficiency assessment standards in second language acquisition research “closing the gap". Studies in Second Language Acquisition, v. 33, p. 339-372, 2011. https:// doi.org/10.1017/S0272263111000015

Recebido em 27/12/2016. Aceito em 29/06/2017. 